\title{
Combined search for dark matter from the Galactic Centre with the ANTARES and IceCube neutrino telescopes
}

\author{
Sara Rebecca Gozzini ${ }^{1, *}$, Nadège Iovine ${ }^{2}$, Juan Antonio Aguilar Sánchez ${ }^{2}$, Sebastian Baur ${ }^{2}$, \\ and Juan de Dios Zornoza Gómez ${ }^{1}$ on behalf of the ANTARES and IceCube Collaborations \\ ${ }^{1}$ IFIC, Instituto de Física Corpuscular, C. José Beltrán Martinez, 2, 46980 Paterna, València, Spain \\ ${ }^{2}$ Université Libre de Bruxelles, Boulevard du Triomphe, CP 230, B-1050 Bruxelles, Belgium
}

\begin{abstract}
The ANTARES and IceCube neutrino telescopes have independently searched for neutrinos from dark matter pair-annihilation in the Galactic Centre, and placed limits on the velocity-averaged WIMP annihilation crosssection $\langle\sigma v\rangle$. To date, the most stringent limits were obtained by the ANTARES neutrino telescope for WIMP masses $>100 \mathrm{GeV} / \mathrm{c}^{2}$, closely followed by the limits of the IceCube experiment for WIMP masses up to $1 \mathrm{TeV} / \mathrm{c}^{2}$. Here we present the sensitivities of a combined search for dark matter in the Galactic Centre using data from both experiments in a WIMP mass range from 100 $\mathrm{GeV} / \mathrm{c}^{2}$ to $1 \mathrm{TeV} / \mathrm{c}^{2}$. This analysis includes IceCube data collected with the complete 86-strings detector from 2012 to 2015 and ANTARES data from 2007 to 2015. The two data sets were combined using a common likelihood framework, and before unblinding the combined sensitivities to $\langle\sigma v\rangle$ are shown.
\end{abstract}

\section{Introduction}

The existence of cold, non-baryonic dark matter is well supported by astrophysical observations and motivates indirect searches for neutrinos from Weakly Interacting Massive Particles (WIMPs) pair annihilations. According to observational evidence, galaxies are embedded in a halo of thermal relic density of dark matter with a high density at the center, providing a target of interest for neutrino telescopes. Limits on WIMP dark matter annihilation crosssection have already been set by the ANTARES [1] and IceCube [2] neutrino experiments independently [3, 4], however, a gain in sensitivity can be achieved in the region where the two single-telescope sensitivities are comparable, for WIMP masses between $100 \mathrm{GeV} / \mathrm{c}^{2}$ and $1 \mathrm{TeV} / \mathrm{c}^{2}$. The purpose of this analysis is to combine the data of the two neutrino detectors by joining the probability density functions (PDFs) to build a two-component likelihood and provide combined limits on the cross section for WIMP pair-annihilation in the Galactic Centre.

\section{Particle phenomenology and cosmology input}

Predictions for dark matter annihilation fluxes strongly rely on its density distribution in the Galaxy and annihilation modes. In WIMP scenarios, dark matter particles of mass $m_{\chi}$

\footnotetext{
*e-mail: sara.gozzini@ific.uv.es
} 
can annihilate into standard model particles including neutrinos as a primary or a secondary product, whose flux at Earth is given by [5]

$$
\frac{d \Phi\left(E_{v}\right)}{d E_{v}}=\frac{1}{4 \pi m_{\chi}^{2}} \frac{\langle\sigma v\rangle}{2} \frac{d N\left(E_{v}\right)}{d E_{v}} J,
$$

where the $J$-factor is defined as the integral of the squared dark matter density along the line of sight. In order to combine the analyses, both ANTARES and IceCube use $J$-factors following the NFW dark matter distribution profile [6] to describe the source morphology. In the evaluation of acceptances, ANTARES uses an integration radius of $30^{\circ}$ around the center of the Galaxy computed with CLUMPY [7], while IceCube assigns a $J$-factor dependent weight to each event computed from its angular separation from the Galactic Centre. The single-annihilation energy distribution $d N\left(E_{v}\right) / d E_{v}$ was obtained with PYTHIA [8] and compared with that previously used in ANTARES analyses [9]. Neutrino oscillations between the source and the detector are taken into account. For the direct annihilation spectra, we consider a $100 \%$ branching ratio for dark matter annihilations into all-flavour neutrinos.

\section{The ANTARES and IceCube neutrino telescopes}

In both the ANTARES and IceCube detectors the reconstruction of the direction, energy and flavor of neutrinos relies on the optical detection of Cherenkov radiation emitted by charged particles produced in the interactions of neutrinos in the surrounding water or ice or in the nearby bedrock.

ANTARES is an underwater Cherenkov telescope located at $2500 \mathrm{~m}$ depth in the Mediterranean sea, about $40 \mathrm{~km}$ offshore from Toulon (France) [1]. The detector consists of an array of 885 optical modules placed along 12 over a surface of $0.1 \mathrm{~km}^{2}$ on the seabed, each line being $450 \mathrm{~m}$ long. ANTARES uses two different track reconstruction algorithms depending on the deposited energy of the events: single-line for events below $100 \mathrm{GeV}$ and multi-line over $100 \mathrm{GeV}$. Here we consider data recorded from 2007 to 2015, over a lifetime of 2101.6 days [3]. The number of events in this sample is 15651 multi-line and 1416 single-line tracks. IceCube is a cubic-kilometer neutrino detector deployed in the South Pole glacier between $1450 \mathrm{~m}$ and $2450 \mathrm{~m}$ depth [2]. The IceCube observatory consists of an array of 5160 digital optical modules attached to vertical strings placed in 86 boreholes. In the centre of the detector, eight strings are deployed in a more compact configuration, forming the DeepCore sub-detector, which extends the detection of neutrinos to energies below $100 \mathrm{GeV}$. For this analysis, we use the IceCube data selection developed in the course of the Galactic Center WIMP search analysis, consisting of 1007 days of track-like events that are compatible with $v_{\mu}$ signatures, taken with the 86-strings configuration between May 15, 2012 and May 18, 2015. The total number of events in this sample is 26976 events.

Although having a smaller instrumented volume than IceCube, ANTARES has the advantage of a privileged view of the Galactic Center, since the Earth is also blocking the main contribution of the atmospheric muon background from the Southern Hemisphere.

\section{Analysis method}

While the atmospheric neutrino background is uniformly distributed in right ascension, the dark-matter annihilation signal would cluster according to the $J$-factor around the position of the Galactic Centre, hence the coordinates of the neutrino events are used as discriminating variables. The algorithm used in this analysis is a binned maximum-likelihood method with 
a two-component mixture model, for five annihilation channels $\left(\tau \tau, \mu^{+} \mu^{-}, b \bar{b}, W^{+} W^{-}, \nu \bar{v}\right)$ assuming a branching ratio of $100 \%$ in each case, and WIMP masses ranging from $100 \mathrm{GeV} / \mathrm{c}^{2}$ to $1 \mathrm{TeV} / c^{2}$. Each experiment contributes to the combined likelihood with their signal and background density distribution (PDFs). In ANTARES the PDFs are distributions of the offset angle $\psi$ between the arrival direction of each neutrino event and the Galactic Center. This holds for all events distributed over multiple lines that could be reconstructed with zenith and azimuth, as shown in Fig. 1 (left). However, for dark matter searches it is crucial to extend the energy threshold as low as possible, so a subset of low-energy events hitting one-line only could still be reconstructed using a dedicated single-line algorithm. These events lack azimuthal information; the PDF is built from the distribution of the cosine zenith difference $\cos \left(\theta_{v}\right)-\cos \left(\theta_{G C}\right)$ as shown in Fig. 1 (right). For IceCube the PDFs are distributions in right ascension and declination, for a band covering $[-\pi / 2, \pi / 2]$ radians in declination and $[-\pi, \pi]$ in right ascension as shown in Fig. 2. A likelihood function is built as the product of Poisson probabilities $\mathcal{P}$ and maximised with respect to the signal-to-background ratio $\mu$

$$
\mathcal{L}(\mu)=\prod_{i=1}^{\text {bins }} \mathcal{P}\left(n_{\text {obs }}^{i} \mid n_{\text {obs }}^{\text {tot }} \cdot f^{i}(\mu)\right)
$$

where $n_{\text {tot }}^{\text {obs }}$ is the total number of background events in the sample, $n_{\text {obs }}^{i}$ the observed number of events in bin $i$, and $f^{i}(\mu)$ is the fraction of events in the bin $i$

$$
f^{i}(\mu)=\mu f_{s}^{i}+(1-\mu) f_{b g}
$$

with $f_{s}$ and $f_{b g}$ being the signal and the background probability densities. The combined likelihood for the two experiments here labelled $A / I$ is $\mathcal{L}_{A+I}(\mu)=\mathcal{L}_{A}\left(\mu_{A}\right) \cdot \mathcal{L}_{I}\left(\mu_{I}\right)$. Each detector has a signal-to-background ratio $\mu_{A / I}=w_{A / I} \mu$ where the weights $w_{A / I}$ are calculated from the relative expected number of signal events in each detector, and the relative number of background events in each sample. The best estimate of the signal fraction is obtained by minimizing $-\log \mathcal{L}_{A+I}(\mu)$ [4]. If this value is consistent with zero, the expected upper limit on the signal fraction, $\mu_{90}$, is estimated by determining the $90 \%$ confidence interval using the Feldman-Cousins approach [10].
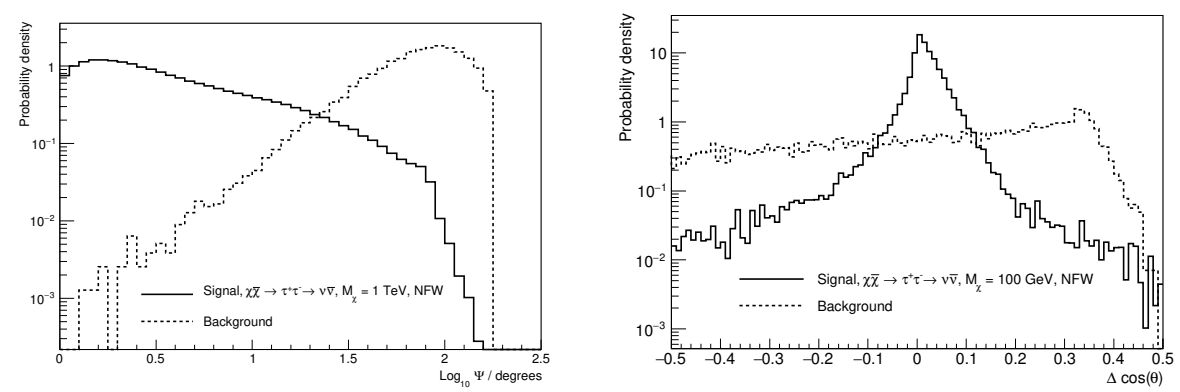

Figure 1. PDFs for ANTARES, multi-line (left) and single-line (right) events. For multi-line events the discriminating variable is the angular offset $\Psi$ between each neutrino track and the Galactic Centre. For single-line events, the discriminating variable is the zenith cosine difference between each event and the Galactic Centre. The signal is represented here for $\tau^{+} \tau^{-}$annihilation channel, NFW profile, $M_{\chi}=1$ $\mathrm{TeV} / \mathrm{c}^{2}$ (multi-line) and $M_{\chi}=100 \mathrm{GeV} / \mathrm{c}^{2}$ (single-line), respectively. 

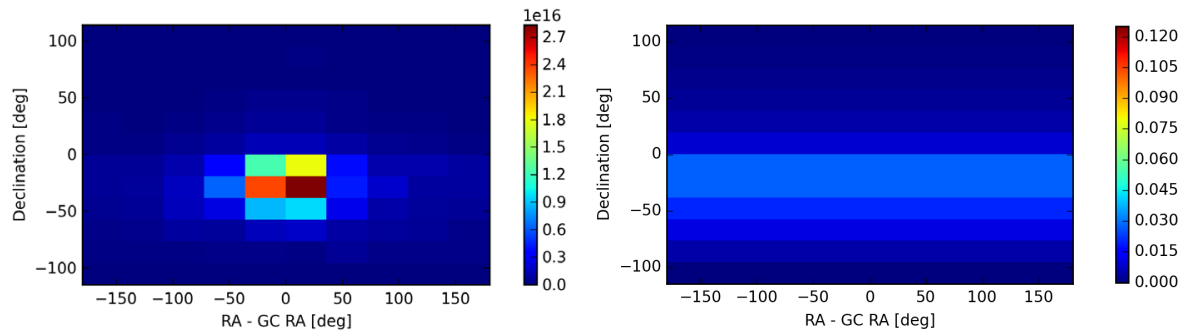

Figure 2. PDFs for IceCube as a function of declination and right ascension for signal (left) and background (right). The signal is for a $\tau^{+} \tau^{-}$annihilation channel, NFW profile and $M_{\chi}=100 \mathrm{GeV} / \mathrm{c}^{2}$.

\section{Results}

We evaluated the sensitivity of the ANTARES data sample with the IceCube binned method presented in Section 4 as a robustness check for the combined analysis. The expected upper limit $\mu_{90}$ on the number of events is shown in Fig. 3 (left) for multi-line and single-line events. The sensitivity to $\langle\sigma v\rangle$ for the combined analysis of IceCube and ANTARES is shown in Fig. 3 (right). The results show an improvement of the sensitivity in the energy range of $65 \mathrm{GeV} / \mathrm{c}^{2}$ to $1 \mathrm{TeV} / \mathrm{c}^{2}$ when compared to the individual results of both ANTARES and IceCube $[3,4]$.
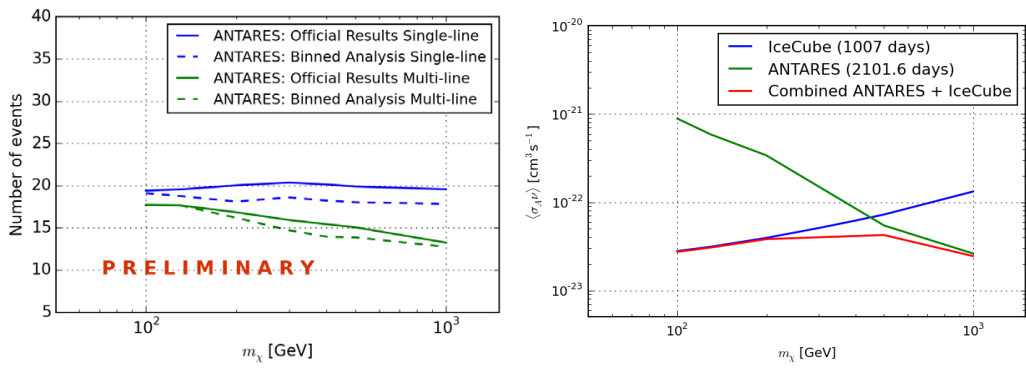

Figure 3. Left: Sensitivity to the number of events for the ANTARES sample when cross-analysed in the IceCube framework, here the $W^{+} W^{-}$channel is shown. Right: Combined sensitivities previously achieved on the thermally averaged cross section for WIMP annihilation for $W^{+} W^{-}$channel.

\section{References}

[1] M. Ageron et al., (ANTARES Collaboration), Nucl.Instrum.Meth. A656, 11-38 (2011)

[2] A. Achterberg et al. (IceCube Collaboration), Astropart.Phys. 26, 155-173 (2006)

[3] A. Albert et al. (ANTARES Collaboration), Phys. Lett. B 769, 249-254 (2017)

[4] M.G. Aartsen et al. (IceCube Collaboration) Eur. Phys. J C77, 627 (2017)

[5] H. Yüksel, S. Horiuchi, J. F. Beacom, and S. Ando, Physical Review D 76, 249 (2007)

[6] J. Navarro, C. Frenk and S. White, The Astrophysical Journal, 462, 563:575 (1996)

[7] M Hütten et al., Computer Physics Communications 235, 336-345 (2019)

[8] T. Sjöstrand et al., Computer Physics Communications, 191, 159-177 (2015)

[9] M. Cirelli et al., JCAP 03051 (2011)

[10] G. J. Feldman and R. D. Cousins, Phys. Rev. D 57, 3873 (1998) 